\title{
Longstanding postoperative fluid collection influences recurrence of pancreatic malignancy
}

\author{
Young Jung Kim ${ }^{1,2}$, Young Koog Cheon ${ }^{1}$, Tae Yoon $\mathrm{Lee}^{1}$, Seong-Hwan Chang ${ }^{3}$, and Mi-Hye Yu
}

${ }^{1}$ Department of Internal Medicine, Konkuk University School of Medicine, Seoul; ${ }^{2}$ Department of Internal Medicine, Chonnam National University Medical School, Gwangju; Departments of ${ }^{3}$ Surgery and

${ }^{4}$ Radiology, Konkuk University School of Medicine, Seoul, Korea
Received: January 26, 2021

Revised : March 2, 2021

Accepted: March 19, 2021

\section{Correspondence to}

Young Koog Cheon, M.D.

Department of Internal

Medicine, Konkuk University

School of Medicine, 120-1

Neungdong-ro, Gwangjin-gu,

Seoul 05030, Korea

Tel: +82-2-2030-7490

Fax: +82-2-2030-5029

E-mail: ykskyoo1@hanmail.net https://orcid.org/0000-0003-

3655-1165
Background/Aims: Postoperative abdominal fluid collection (PAFC) is a frequent complication of pancreatobiliary cancer surgery. The effects of the existence and duration of PAFC are not well known. This study aimed to assess the effects of PAFC on patient prognosis after surgery for pancreatobiliary adenocarcinoma and the association of longstanding PAFC with the recurrence of pancreatic cancer.

Methods: We retrospectively analyzed the data of 194 consecutive patients with pancreatobiliary adenocarcinoma who underwent curative operations from $\mathrm{Au}$ gust 2005 to December 2019. The presence of PAFC was assessed using computed tomography within a week of surgery; PAFC lasting > 4 weeks was defined as longstanding PAFC.

Results: Among 194 patients, PAFC occurred in 165 (85.1\%), and 74 of these had longstanding PAFC. The recurrence rate of pancreatobiliary adenocarcinoma was significantly higher in patients with longstanding PAFC than in patients with non-longstanding PAFC $(p=0.025)$. Recurrence was also significantly associated with high $\mathrm{T}$ stage $\left(\mathrm{T}_{3}, \mathrm{~T}_{4} ; p=0.040\right)$, lymph node involvement $(p<0.001)$, perineural invasion $(p<0.006)$, and non-receipt of adjuvant chemotherapy $(p=0.025)$. Longstanding PAFC was significantly associated with the recurrence of pancreatic adenocarcinoma $(p=0.016)$. However, cancer-specific survival was related to neither the presence nor the duration of PAFC.

Conclusions: The presence of longstanding PAFC was associated with the recurrence of pancreatic adenocarcinoma. However, a larger prospective study is necessary to confirm the findings.

Keywords: Pancreatic neoplasms; Biliary tract neoplasms; Recurrence; Survival; Ascitic fluid

\section{INTRODUCTION}

Pancreatobiliary adenocarcinoma can occur in the pancreas, bile duct, ampulla of Vater, or gallbladder. Surgery with curative intent results in long-term survival, except in cases where the cancer has progressed or other problems occur. Postoperative complications frequently occur in patients with pancreatobiliary adenocarcinoma. The overall morbidity rate is $46 \%$ in patients who un- dergo pancreatic resection, with surgical and nonsurgical complications occurring in $31 \%$ and $27 \%$ of patients, respectively [1]. Postoperative abdominal fluid collection (PAFC) is a frequent nonsurgical complication, occurring in $4 \%$ to $21 \%$ of patients after total or partial organ resection, biliary or intestinal bypass, gastrointestinal reconstruction, or solid organ transplantation [1].

Despite developments in imaging modalities, surgical techniques, perioperative management, and chemo- 
therapy, pancreatobiliary adenocarcinoma has a poor prognosis, which may be due to the high incidence of recurrence after surgery. The 5-year survival rate after surgery for pancreatic adenocarcinoma is $8 \%$ to $10 \%[2-4]$, in contrast to $44 \%$ to $70 \%$ for intrahepatic cholangiocarcinoma [5]. Predictive factors for postoperative complications in pancreatobiliary adenocarcinoma have been studied $[6,7]$. Although the effects of postoperative complications on long-term survival have been investigated $[8,9]$, little is known about the effects of the presence and duration of PAFC on long-term survival and recurrence of pancreatobiliary adenocarcinoma after surgery.

This study aimed to determine the incidence of PAFC and to assess its effects on patient prognosis after surgery for pancreatobiliary adenocarcinoma, with a particular focus on pancreatic cancer.

\section{METHODS}

\section{Patients and methods}

From August 2005 to December 2019, 297 patients with pancreatobiliary malignancy underwent surgery with a curative intent at our hospital. Of 297 patients, 194 with histologically proven adenocarcinoma were included in this retrospective cohort study. Their medical records, computed tomography (CT) findings, surgical procedures, pathological findings, adjuvant chemotherapy data, and survival outcomes were retrospectively reviewed. We classified cancers of pancreatobiliary origin as ampulla of Vater cancer, gallbladder cancer, pancreatic cancer, and cholangiocarcinoma (e.g., Klatskin tumor, extrahepatic and intrahepatic cholangiocarcinoma).

Regarding pathological findings, we assessed the pathological tumor-node-metastasis (pTNM)-stage, presence of microscopically remnant disease, perineural invasion, total count of resected lymph nodes, and total count of positive lymph nodes. The $\mathrm{pT}$ stages of $\mathrm{T}_{3}$ and $\mathrm{T}_{4}$ were classified as high and those of $\mathrm{T}_{1}$ and $\mathrm{T} 2 \mathrm{as}$ low. The TNM stage was evaluated based on the American Joint Committee on Cancer (AJCC) Cancer Staging Manual, 7th edition [10].

Recurrence was defined as confirmation of the presence of a lesion by radiology or biopsy. Local recurrence was defined as reappearance of a tumorous lesion at the anastomosis site or around the PAFC based on imaging findings.

\section{Postoperative abdominal fluid collection}

PAFC was defined as a collection of fluid within a diameter $\geq 3 \mathrm{~cm}$, as demonstrated by CT $[5,11]$. The presence or absence of PAFC on CT was reviewed by radiologists. The duration index date was defined according to the postoperative day. At our hospital, CT is performed routinely to assess postoperative complications within 1 week after surgery. A second CT examination is performed at 4 weeks postoperatively to determine whether the postoperative complications, including PAFC, have improved. Based on this CT schedule, longstanding PAFC was defined as PAFC that did not regress spontaneously and was detected by CT or ultrasound for $>4$ weeks after surgery. We excluded patients who did not undergo CT examinations at 4 weeks postoperatively. Patients with preoperative ascites and those receiving ambulatory peritoneal dialysis were excluded due to the difficulty of identifying PAFC in such patients.

\section{Follow-up}

All follow-up data, including the date of last follow-up, vital status, cancer recurrence, and survival, were obtained by contacting doctors and hospitals and from registry medical records. Survival duration was measured from the date of surgery until death or the date of the last hospital visit. Patients lost to follow-up were also excluded.

\section{Statistical analysis}

The recurrence of pancreatobiliary adenocarcinoma was the primary outcome measure. Categorical variables were expressed as frequencies and percentages, and continuous variables were expressed as mean \pm standard deviation (or ranges). The variables were compared using the chi-square test. Variables with $p$ values $<0.2$ were entered into a logistic regression model for multivariate analyses. Cancer-specific survival (CSS) was considered as a secondary outcome and was calculated using the Kaplan-Meier method. Variables with $p$ values $<0.2$ were entered into a Cox proportional-hazards regression model for multivariate analyses. Patients who died due to surgical complications or causes other than cancer were excluded.

Statistical analysis was conducted using SPSS statistical software version 24.0 (IBM Co., Armonk, NY, USA). $p$ values $<0.05$ were regarded as statistically significant. 


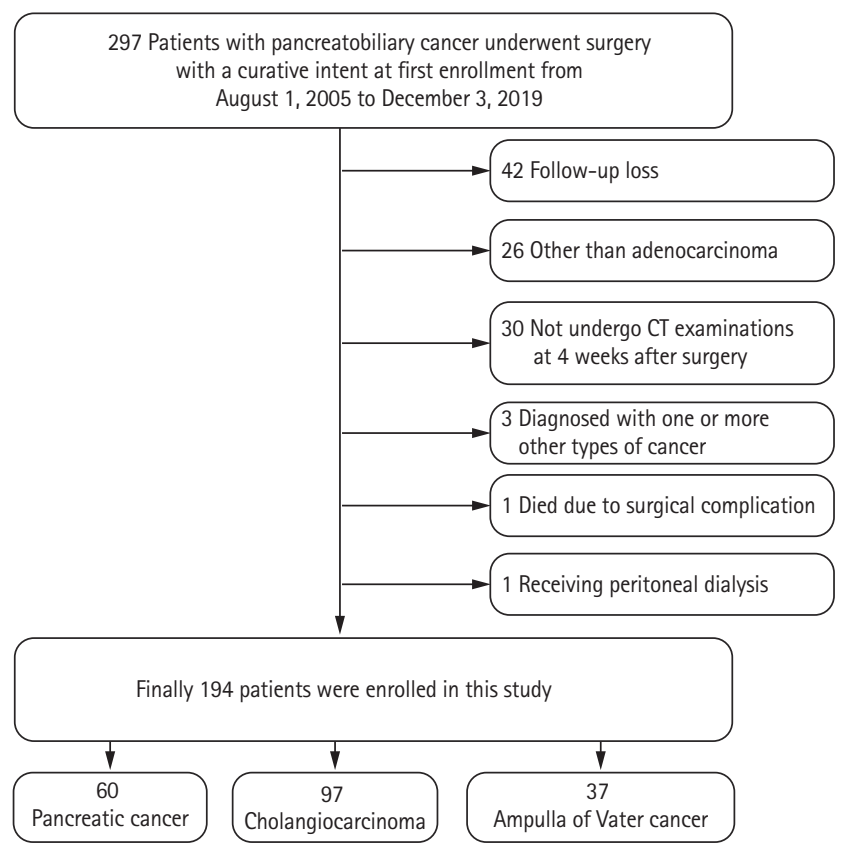

Figure 1. Flow diagram of enrolled patients. CT, computed tomography.

This study was performed according to the principles of the Declaration of Helsinki. The Institutional Review Board of Konkuk University Medical Center approved this study (approval number: KUH1010922). The requirement of informed patient consent was waived by the Institutional Review Board due to the retrospective nature of the study.

\section{RESULTS}

\section{Baseline characteristics}

In total, 297 patients with pancreatobiliary duct cancer underwent surgery with curative intent; 194 of these were eligible for the present study. Of these, 103 patients were excluded for the following reasons: 42 patients were lost to follow-up, 26 patients were diagnosed with conditions other than adenocarcinoma (e.g., neuroendocrine tumor, pancreatic serous cystadenoma, solitary pseudopapillary tumor, intraductal papillary mucinous neoplasms, and benign pathology), 30 patients did not undergo CT examinations at 4 weeks after surgery, three patients were diagnosed with one or more other types of cancer, one patient died due to surgical complication, and one patient was receiving peritoneal dialysis (Fig. 1). The baseline clinical data are summarized in Table 1. The mean age of the patients was $68 \pm 9.7$ years. One hundred and four (53.6\%) patients were men and 90 (46.4\%) were women. Sixty-six (34.0\%) patients had hypertension, 22 (11.3\%) had diabetes mellitus, and 52 (26.8\%) had dyslipidemia. The mean follow-up period was $37 \pm 28.9$ months. The most common tumor type was cholangiocarcinoma (97 patients, 50.0\%). Fifty-seven patients had distal cholangiocarcinoma, 16 had Klatskin tumor, and 24 had intrahepatic cholangiocarcinoma. Sixty (30.9\%) patients had pancreatic cancer and 37 (19.1\%) had ampulla of Vater cancer. The resection margin status was positive in $21(10.8 \%)$ patients. PAFC was detected by CT in $165(85.1 \%)$ patients and was longstanding (> 4 weeks) in $74(38.1 \%)$ of these patients. Of the 74 patients with longstanding PAFC, the fluid collection improved with medical treatment in only 22 patients. In the remaining 52 patients, PAFC did not disappear during the follow-up period; however, no surgical intervention was attempted because complicated fluid collections did not occur in this group. Cancer recurred during the follow-up period in 114 (58.8\%) patients: local recurrence in 92 (47.4\%) patients and metastatic recurrence in 22 (11.3\%) patients. Seventy-seven (39.7\%) patients died due to cancer recurrence. The surgical approach for resection of pancreatobiliary cancer and the prevalence of longstanding PAFC are summarized in Table 2.

\section{Risk factors for recurrence}

In univariate analyses, the recurrence rates were 58.8\% $(\mathrm{n}=97)$ in patients with PAFC and 58.6\% $(\mathrm{n}=17)$ in patients without PAFC $(p=0.987)$. The recurrence rate was significantly higher in patients with longstanding PAFC (68.9\%, $\mathrm{n}=51 ; \mathrm{p}=0.025)$ than in patients with non-longstanding PAFC (50.5\%, $n=46 ; p=0.025)$. Fig. 2 shows a local recurrence in a patient with longstanding PAFC. Recurrence was also significantly associated with high T stage ( $\left.\mathrm{T}_{3}, \mathrm{~T}_{4} ; p=0.040\right)$, lymph node involvement $(p<$ $0.001)$, perineural invasion $(p<0.006)$, and non-receipt of adjuvant chemotherapy ( $p=0.025)$. Age ( $>70$ years), sex, resection margin status, and presence of PAFC were not associated significantly with recurrence (Table 3).

In the multivariate analysis, the recurrence rate was associated with lymph node involvement $(p=0.003)$ and longstanding PAFC $(p=0.020)$. No other variable was as- 
Table 1. Clinicopathologic characteristics of 194 patients with pancreatobiliary adenocarcinoma

\begin{tabular}{|c|c|}
\hline Characteristic & Value \\
\hline \multicolumn{2}{|l|}{ Patient variable } \\
\hline Age, yr & $68 \pm 9.7$ \\
\hline \multicolumn{2}{|l|}{ Sex } \\
\hline Male & $104(53.6)$ \\
\hline Female & $90(46.4)$ \\
\hline \multicolumn{2}{|l|}{ Underlying disease } \\
\hline Hypertension & $66(34.0)$ \\
\hline Diabetes mellitus & $22(11.34)$ \\
\hline Dyslipidemia & $52(26.8)$ \\
\hline Follow-up period, mo & $37 \pm 28.9$ \\
\hline \multicolumn{2}{|l|}{ Tumor type } \\
\hline Pancreatic cancer & $60(30.9)$ \\
\hline Cholangiocarcinoma & $97(50.0)$ \\
\hline Ampulla of Vater cancer & $37(19.1)$ \\
\hline \multicolumn{2}{|l|}{ Pathologic variable } \\
\hline \multicolumn{2}{|l|}{ p'-stage } \\
\hline $\mathrm{T} 1, \mathrm{~T}_{2}$ & $91(46.9)$ \\
\hline $\mathrm{T}_{3}, \mathrm{~T}_{4}$ & $103(53.1)$ \\
\hline \multicolumn{2}{|l|}{ Lymph node metastasis } \\
\hline Present & $86(44 \cdot 3)$ \\
\hline Not present & $108(55 \cdot 7)$ \\
\hline \multicolumn{2}{|l|}{ Perineural invasion } \\
\hline Present & $117(60.3)$ \\
\hline Not present & $77(39 \cdot 7)$ \\
\hline \multicolumn{2}{|l|}{ Resection margin status } \\
\hline Positive & $21(10.8)$ \\
\hline Negative & $173(89.2)$ \\
\hline \multicolumn{2}{|l|}{ Postoperative fluid collection } \\
\hline Present & $165(85.1)$ \\
\hline Not present & $29(14.9)$ \\
\hline \multicolumn{2}{|l|}{ Postoperative fluid duration } \\
\hline Less than 4 weeks & $91(46.9)$ \\
\hline More than 4 weeks & $74(38.1)$ \\
\hline Received adjuvant chemotherapy & $120(61.9)$ \\
\hline Cancer recurrence & $114(58.8)$ \\
\hline Local recurrence & $92(47 \cdot 4)$ \\
\hline Died due to cancer recurrence & $77(39.7)$ \\
\hline
\end{tabular}

Values are presented as mean \pm SD or number (\%). pT, pathologic T.
Table 2. Surgical approach to pancreatobiliary cancer and the prevalence of longstanding PAFC

\begin{tabular}{lcc}
\hline Surgical method & Number & $\begin{array}{c}\text { Longstanding } \\
\text { PAFC }\end{array}$ \\
\hline Pancreatic cancer & 18 & 13 \\
\hline Distal pancreatectomy & 16 & 6 \\
\hline Whipple's operation & 24 & 6 \\
\hline PPPD & 2 & 0 \\
\hline Total pancreatectomy & 13 & 5 \\
Cholangiocarcinoma & 11 & 7 \\
Bile duct radical resection & 13 & 8 \\
\hline Hemihepatectomy with HJS & 7 & 2 \\
\hline Right lobectomy & 17 & 6 \\
\hline Left lobectomy & 36 & 9 \\
\hline Partial hepatectomy & & 8 \\
\hline Whipple's operation & 16 & 16 \\
\hline PPPD & 58 & \\
\hline Ampulla of Vater cancer & & \\
\hline Whipple's operation & & \\
\hline PPPD & & \\
\hline PAF & & \\
\hline
\end{tabular}

PAFC, postoperative abdominal fluid collection; PPPD, pylorus-preserving pancreaticoduodenectomy; HJS, hepaticojejunostomy.

sociated with the recurrence rates (Table 4).

The presence of PAFC was not significantly related to recurrence of any subtype of pancreatobiliary adenocarcinoma. However, longstanding PAFC was significantly related to recurrence of pancreatic adenocarcinoma $(p=$ o.016) (Table 5) in univariate analyses.

\section{Risk factors for poor CSS}

In univariate analyses, poor CSS was significantly associated with a high $\mathrm{T}$ stage ( $\left.\mathrm{T}_{3}, \mathrm{~T}_{4} ; p=0.001\right)$, lymph node involvement $(p<0.001)$, perineural invasion $(p=0.004)$, and non-receipt of adjuvant chemotherapy $(p=0.002)$. Age ( $>70$ years), sex, and resection margin status were not significantly associated with poor CSS (Table 6).

In the multivariate analysis, poor CSS was associated with a high $\mathrm{T}$ stage $\left(\mathrm{T}_{3}, \mathrm{~T}_{4} ; p=0.042\right)$ and lymph node involvement only $(p<0.001$ ) (Table 7). However, in both univariate and multivariate analyses of patients with pancreatic adenocarcinoma, no variable was associated with poor CSS. 

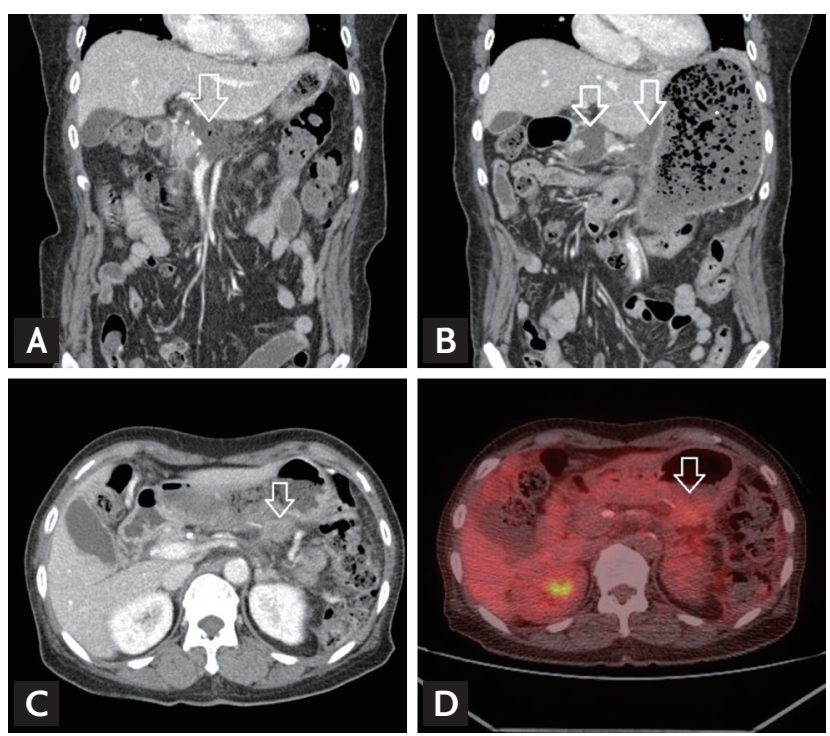

Figure 2. Computed tomography (CT) findings of cancer recurrence at the operative bed in a patient with longstanding postoperative abdominal fluid collection. (A) Seven days after distal pancreatectomy, fluid collection (arrow) at the operative bed. (B) Four weeks after distal pancreatectomy, sustained fluid collection (arrows) at the operative bed. (C) Ten months after distal pancreatectomy, recurrence of cancer in the operative bed (arrow). (D) Positron emission tomography-computed tomography showed focal hypermetabolic lesion (arrow) at the operative bed.

\section{DISCUSSION}

This study demonstrated a significant association between the duration of PAFC and tumor recurrence after surgery for pancreatobiliary adenocarcinoma. Our results suggest that the recurrence rate was higher in patients with longstanding PAFC than in patients with non-longstanding PAFC.

PAFC occurs frequently in patients with pancreatobiliary adenocarcinoma. Although other postoperative complications, such as pancreatic fistula, delayed gastric emptying, abdominal bleeding, and abscess, have been investigated extensively, few studies have evaluated the impact of longstanding PAFC on tumor recurrence and long-term survival [1]. Therefore, we analyzed the clinical impact of longstanding PAFC on recurrence and survival after curative resection of pancreatobiliary adenocarcinoma. We excluded patients with complicated postoperative fluid collection (i.e., those with fever, bile leak, or pancreatic fistula). This condition increases the incidence of PAFC [11-14] and necessitates an additional drainage procedure [14].

In this study, $85.1 \%$ of patients developed PAFC after surgery for pancreatobiliary adenocarcinoma. PAFC resolved spontaneously (without intervention) in $55.2 \%$ of

Table 3. Univariate chi-square analysis of factors predictive of recurrence in pancreatobiliary adenocarcinoma

\begin{tabular}{|c|c|c|c|}
\hline \multirow{2}{*}{ Characteristic } & \multicolumn{3}{|c|}{ Univariate } \\
\hline & HR & $95 \% \mathrm{CI}$ & $p$ value \\
\hline Male sex & 0.927 & $0.523-1.644$ & 0.795 \\
\hline Age $>70$ years & 0.851 & $0.480-1.510$ & 0.581 \\
\hline \multicolumn{4}{|l|}{ pT-stage } \\
\hline $\mathrm{T}_{3}, \mathrm{~T}_{4}$ & 1.901 & $1.065-3.391$ & $0.040^{\mathrm{a}}$ \\
\hline Lymph node involvement, yes & 3.727 & $2.003-6.934$ & $<0.001^{\mathrm{a}}$ \\
\hline Perineural invasion, yes & 2.278 & $1.263-4.109$ & $0.006^{\mathrm{a}}$ \\
\hline Resection margin, positive & 1.460 & $0.561-3.798$ & 0.436 \\
\hline Postoperative fluid collection, yes & 1.007 & $0.45^{2-2.244}$ & 0.987 \\
\hline \multicolumn{4}{|c|}{ Postoperative fluid collection duration } \\
\hline More than 4 weeks & 2.006 & $1.091-3.688$ & $0.025^{\mathrm{a}}$ \\
\hline Adjuvant chemotherapy, yes & 0.510 & $0.283-0.921$ & $0.025^{\mathrm{a}}$ \\
\hline
\end{tabular}

HR, hazard ratio; CI, confidence interval; pT, pathologic T.

${ }^{a} p<0.05$ 
Kim YJ, et al. PAFC and recurrence of pancreatic cancer

Table 4. Logistic regression analysis of factors predictive of recurrence in pancreatobiliary adenocarcinoma

\begin{tabular}{|c|c|c|c|}
\hline \multirow{2}{*}{ Characteristic } & \multicolumn{3}{|c|}{ Multivariate } \\
\hline & $\mathrm{HR}$ & $95 \% \mathrm{CI}$ & $p$ value \\
\hline \multicolumn{4}{|l|}{ p'T-stage } \\
\hline $\mathrm{T}_{3}, \mathrm{~T}_{4}$ & 1.405 & $0.714-2.761$ & 0.325 \\
\hline Lymph node involvement, yes & 2.966 & $1.454-6.052$ & $0.003^{\mathrm{a}}$ \\
\hline Perineural invasion, yes & 1.612 & $0.813-3.196$ & 0.172 \\
\hline \multicolumn{4}{|c|}{ Postoperative fluid collection duration } \\
\hline More than 4 weeks & 2.227 & $1.137-4.363$ & $0.020^{\mathrm{a}}$ \\
\hline Adjuvant chemotherapy, yes & 1.091 & $0.539-2.211$ & 0.808 \\
\hline
\end{tabular}

HR, hazard ratio; CI, confidence interval; pT, pathologic T.

${ }^{\mathrm{a}} p<0.05$.

Table 5. Univariate chi-square analysis of factors predictive of recurrence of pancreatic cancer, cholangiocarcinoma, and ampulla of Vater cancer

\begin{tabular}{|c|c|c|c|c|c|c|c|c|c|}
\hline \multirow{2}{*}{ Characteristic } & \multicolumn{3}{|c|}{ Pancreatic cancer } & \multicolumn{3}{|c|}{ Cholangiocarcinoma } & \multicolumn{3}{|c|}{ Ampulla of Vater cancer } \\
\hline & $\mathrm{HR}$ & $95 \% \mathrm{CI}$ & $p$ value & $\mathrm{HR}$ & $95 \% \mathrm{CI}$ & $p$ value & HR & $95 \%$ CI & $p$ value \\
\hline Male sex & 0.902 & $0.303-2.689$ & 0.853 & 0.705 & $0.304-1.633$ & 0.414 & 1.800 & $0.476-6.812$ & 0.385 \\
\hline Age $>70$ years & 0.815 & $0.275^{-2.410}$ & 0.711 & 0.903 & $0.399-2.042$ & 0.807 & 0.875 & $0.235-3.257$ & 0.842 \\
\hline \multicolumn{10}{|l|}{ p'-stage } \\
\hline $\mathrm{T}_{3}, \mathrm{~T}_{4}$ & 1.750 & $0.414-7.399$ & 0.704 & 0.847 & $0.365-1.965$ & 0.699 & 18.000 & $3.403-95.204$ & $<0.001^{\mathrm{a}}$ \\
\hline $\begin{array}{l}\text { Lymph node } \\
\text { involvement, yes }\end{array}$ & 2.636 & $0.862-8.064$ & 0.085 & 2.719 & $1.098-6.730$ & $0.028^{a}$ & 15.000 & $2.521-89.235$ & $0.001^{\mathrm{a}}$ \\
\hline $\begin{array}{l}\text { Perineural } \\
\text { invasion, yes }\end{array}$ & 2.429 & $0.668-8.835$ & 0.304 & 0.793 & $0.343-1.833$ & 0.588 & 42.000 & $4.316-408.680$ & $<0.001^{\mathrm{a}}$ \\
\hline $\begin{array}{l}\text { Resection margin, } \\
\text { positive }\end{array}$ & 0.459 & $0.084-2.516$ & 0.653 & 1.735 & $0.502-5.990$ & 0.380 & & & 0.405 \\
\hline $\begin{array}{l}\text { Postoperative fluid } \\
\text { collection, yes }\end{array}$ & 1.179 & $0.301-4.619$ & 1.000 & 0.546 & $0.135^{-2.205}$ & 0.519 & 1.912 & $0.319-11.471$ & 0.677 \\
\hline \multicolumn{10}{|l|}{$\begin{array}{l}\text { Postoperative fluid } \\
\text { collection duration }\end{array}$} \\
\hline More than 4 weeks & $4 \cdot 421$ & $1.255^{-15.573}$ & $0.016^{\mathrm{a}}$ & 1.593 & $0.676-3.754$ & 0.285 & 1.071 & $0.264-4 \cdot 341$ & 0.923 \\
\hline $\begin{array}{l}\text { Adjuvant } \\
\text { chemotherapy, yes }\end{array}$ & 2.250 & $0.431-11.758$ & 0.471 & 0.553 & $0.241-1.267$ & 0.159 & 0.257 & $0.062-1.067$ & 0.056 \\
\hline
\end{tabular}

HR, hazard ratio; CI, confidence interval; $\mathrm{pT}$, pathologic T.

a $p<0.05$.

these patients within 4 weeks; however, $44.8 \%$ of these patients had longstanding ( $>4$ weeks) PAFC. The reported incidence of PAFC after pancreatobiliary surgery is approximately $21 \%$ [1], significantly lower than the incidence observed in this study. This difference occurred because our patients had pancreatobiliary adenocarcinoma, whereas previous studies involved patients with benign pancreatobiliary diseases. Therefore, patients involved in this study had undergone more extensive surgeries than those examined in previous studies $[1,2,15]$. Previous studies used abdominal ultrasound to screen asymptomatic patients, which could underestimate fluid collection rates compared to CT [1]. Furthermore, imaging tests were conducted only in patients with symp- 
Table 6. Kaplan-Meier analysis of cancer-specific survival of patients with pancreatobiliary adenocarcinoma after surgery

\begin{tabular}{|c|c|}
\hline Characteristic & $p$ value \\
\hline Male sex & 0.785 \\
\hline Age $>70$ years & 0.919 \\
\hline \multicolumn{2}{|l|}{ p'T-stage } \\
\hline $\mathrm{T}_{3}, \mathrm{~T}_{4}$ & $0.001^{\mathrm{a}}$ \\
\hline Lymph node involvement, yes & $<0.001^{\mathrm{a}}$ \\
\hline Perineural invasion, yes & $0.004^{\mathrm{a}}$ \\
\hline Resection margin, positive & 0.597 \\
\hline Postoperative fluid collection, yes & 0.512 \\
\hline \multicolumn{2}{|c|}{ Postoperative fluid collection duration } \\
\hline More than 4 weeks & 0.192 \\
\hline Adjuvant chemotherapy, yes & $0.002^{\mathrm{a}}$ \\
\hline
\end{tabular}

pT, pathologic T.

${ }^{\mathrm{a}} \mathrm{p}<0.05$.

Table 7. Cox regression analysis of cancer-specific survival of patients with pancreatobiliary adenocarcinoma after surgery

\begin{tabular}{lrrr}
\hline \multirow{2}{*}{ Characteristic } & \multicolumn{3}{c}{ Multivariate } \\
\cline { 2 - 4 } & HR & $95 \%$ CI & p value \\
\hline p'-stage & & & \\
$\quad \mathrm{T}_{3}, \mathrm{~T}_{4}$ & 1.687 & $1.018-2.795$ & $0.042^{\mathrm{a}}$ \\
$\begin{array}{l}\text { Lymph node } \\
\text { involvement, yes }\end{array}$ & 2.563 & $1.571-4.181$ & $<0.001^{\mathrm{a}}$ \\
\hline
\end{tabular}

HR, hazard ratio; CI, confidence interval; $\mathrm{pT}$, pathologic T. ${ }^{a} p<0.05$.

toms suggesting abdominal complications [16].

Postoperative complications are associated with decreased survival in patients with pancreatobiliary malignancies $[8,17]$. A recent meta-analysis showed that postoperative complications carry a hazard ratio of 1.28 for shorter overall survival [18]. However, most previous studies evaluated the detrimental effects of major postoperative complications, rather than their relationships to tumor recurrence. In this study, no significant difference was noted in the recurrence rate between patients with and without PAFC (58.8\% and 58.6\%, respectively). However, the recurrence rate was significantly higher in patients with longstanding PAFC and reached significance in those with pancreatobiliary adenocarcinoma.
In a subgroup analysis, longstanding PAFC was significantly associated with the recurrence of pancreatic adenocarcinoma. However, there was no significant relationship between the recurrence of the other two types of biliary cancer and longstanding PAFC.

The occurrence of major surgical complications leads to transiently damaged cellular immunity, which may result in early cancer recurrence and reduced survival $[6,19]$. During surgery for pancreatobiliary malignancy, the bile and pancreatic ducts are resected. In general, surgery for pancreatic cancer tends to involve a more extensive resection than that for other types of biliary cancer. Minimal residual cancer cells from the duct fluids enter the postoperative fluid collection and are not absorbed rapidly; these cells remain microscopically in the abdominal cavity for a long time and could induce local tumor recurrence. Bile leakage indicates a poor outcome in patients with adenocarcinoma of the pancreatic duct [20].

High pathologic stage, lymph node metastasis, perineural invasion, and non-receipt of adjuvant chemotherapy were associated with the recurrence of pancreatobiliary adenocarcinoma and poor CSS in this study. These findings are consistent with those of previous investigations. However, in patients with pancreatic adenocarcinoma, only longstanding PAFC was associated with the recurrence of cancer. No variable was associated with poor CSS. This was probably due to a small sample size. The prognosis of patients after surgical resection of adenocarcinoma is associated with surgery-related factors (resection margin status and postoperative complications), treatment-related factors (receipt of adjuvant or neoadjuvant chemotherapy), and various tumor-specific factors (tumor differentiation, TNM stage, lymphovascular invasion, and lymph node metastasis) [21].

The minimization of complications preserves physiologic function, ensuring that appropriate adjuvant therapy can be administered in a timely manner [22] Approximately $61.9 \%$ of the patients included in this study received adjuvant chemotherapy within 4 weeks and achieved a better survival rate $(p=0.002)$ and a lower local recurrence rate $(p=0.025)$ than patients who did not receive adjuvant chemotherapy. Patients with major postoperative complications (including PAFC) may require a longer time for physiologic function to stabilize sufficiently for adjuvant chemotherapy. This additional 
time may allow for the propagation and dissemination of microscopic residual tumors, especially in the presence of immunologic impairment [23].

A negative resection margin after pancreatoduodenectomy for pancreatic or periampullary adenocarcinoma is related to improved CSS [11]. A positive resection margin has been related to poor long-term survival, likely due to a greater tumor burden and possibly more aggressive disease $[24,25]$. However, the resection margin status was not substantially related to recurrence or poor CSS in this study. Patients with positive margins routinely receive chemotherapy at our hospital. Thus, the margin status likely did not affect CSS in our study.

This study was retrospective; therefore, cytologic examination of fluid collections was not performed. In a prospective study, it is recommended that cytological examinations be performed. In addition, this study had a small sample size. Therefore, a larger prospective cohort study is needed to clarify the prognostic role of longstanding PAFC in the recurrence of pancreatic adenocarcinoma.

In conclusion, the incidence of PAFC in this study was $85.1 \%$. The recurrence rate of pancreatobiliary adenocarcinoma was higher (68.9\%) among patients with longstanding ( $>4$ weeks) PAFC than among those whose PAFC resolved within 4 weeks. In a subgroup analysis, the recurrence of pancreatic adenocarcinoma was significantly related to longstanding PAFC. Therefore, patients who have longstanding PAFC after pancreatic cancer surgery require more careful observation. If longstanding PAFC is associated with the recurrence of pancreatic adenocarcinoma, the recurrence rate should be reduced by more aggressive treatments in these patients.

\section{KEY MESSAGE}

1. Postoperative abdominal fluid collection (PAFC) is a frequent complication after surgery for pancreatobiliary adenocarcinoma. We investigated the correlation between the duration of PAFC and tumor recurrence after surgery.

2. The recurrence rate of pancreatic adenocarcinoma was higher in patients with longstanding PAFC (> 4 weeks) than in patients with non-longstanding PAFC.

3. Individuals with longstanding PAFC after pancreatic cancer surgery require more careful observation for cancer recurrence.

\section{Conflict of interest}

No potential conflict of interest relevant to this article was reported.

\section{Acknowledgments}

We would like to express our great appreciation to all professors of the Konkuk University Medical Center, who provided helpful comments and suggestions.

\section{REFERENCES}

1. Sierzega M, Kulig P, Kolodziejczyk P, Kulig J. Natural history of intra-abdominal fluid collections following pancreatic surgery. J Gastrointest Surg 2013;17:1406-1413.

2. Kuhlmann KF, de Castro SM, Wesseling JG, et al. Surgical treatment of pancreatic adenocarcinoma; actual survival and prognostic factors in 343 patients. Eur J Cancer 2004;40:549-558.

3. Smeenk HG, Tran TC, Erdmann J, van Eijck CH, Jeekel J. Survival after surgical management of pancreatic adenocarcinoma: does curative and radical surgery truly exist? Langenbecks Arch Surg 2005;390:94-103.

4. Sharma C, Eltawil KM, Renfrew PD, Walsh MJ, Molinari M. Advances in diagnosis, treatment and palliation of pancreatic carcinoma: 1990-2010. World J Gastroenterol 2011;17:867-897.

5. Ercolani G, Vetrone G, Grazi GL, et al. Intrahepatic cholangiocarcinoma: primary liver resection and aggressive multimodal treatment of recurrence significantly prolong survival. Ann Surg 2010;252:107-114.

6. Khuri SF, Henderson WG, DePalma RG, et al. Determinants of long-term survival after major surgery and the adverse effect of postoperative complications. Ann Surg 2005;242:326-341.

7. Aoki S, Miyata H, Konno H, et al. Risk factors of serious postoperative complications after pancreaticoduodenectomy and risk calculators for predicting postoperative complications: a nationwide study of 17,564 patients in 
Japan. J Hepatobiliary Pancreat Sci 2017;24:243-251.

8. Aahlin EK, Olsen F, Uleberg B, Jacobsen BK, Lassen K. Major postoperative complications are associated with impaired long-term survival after gastro-esophageal and pancreatic cancer surgery: a complete national cohort study. BMC Surg 2016;16:32.

9. Kasahara N, Noda H, Kakizawa N, et al. A lack of postoperative complications after pancreatectomy contributes to the long-term survival of patients with pancreatic cancer. Pancreatology 2019;19:686-694.

10. Edge SB, Compton CC. The American Joint Committee on Cancer: the 7th edition of the AJCC cancer staging manual and the future of TNM. Ann Surg Oncol 2010;17:1471-1474.

11. Cullen JJ, Sarr MG, Ilstrup DM. Pancreatic anastomotic leak after pancreaticoduodenectomy: incidence, significance, and management. Am J Surg 1994;168:295-298.

12. Lin JW, Cameron JL, Yeo CJ, Riall TS, Lillemoe KD. Risk factors and outcomes in postpancreaticoduodenectomy pancreaticocutaneous fistula. J Gastrointest Surg 2004;8:951-959.

13. Munoz-Bongrand N, Sauvanet A, Denys A, Sibert A, Vilgrain V, Belghiti J. Conservative management of pancreatic fistula after pancreaticoduodenectomy with pancreaticogastrostomy. J Am Coll Surg 2004;199:198-203.

14. Pratt W, Maithel SK, Vanounou T, Callery MP, Vollmer CM Jr. Postoperative pancreatic fistulas are not equivalent after proximal, distal, and central pancreatectomy. J Gastrointest Surg 2006;10:1264-1278

15. Tjaden C, Hinz U, Hassenpflug M, et al. Fluid collection after distal pancreatectomy: a frequent finding. HPB (Oxford) 2016;18:35-40.

16. Bassi C, Falconi M, Salvia R, Mascetta G, Molinari E, Pederzoli P. Management of complications after pancreaticoduodenectomy in a high volume centre: results on 150 consecutive patients. Dig Surg 2001;18:453-457.

17. van der Gaag NA, Harmsen K, Eshuis WJ, Busch ORC, van Gulik TM, Gouma DJ. Pancreatoduodenectomy associated complications influence cancer recurrence and time interval to death. Eur J Surg Oncol 2014;40:551-558.

18. Pucher PH, Aggarwal R, Qurashi M, Darzi A. Meta-analysis of the effect of postoperative in-hospital morbidity on long-term patient survival. Br J Surg 2014;101:1499-1508.

19. Cho JY, Han HS, Yoon YS, Hwang DW, Jung K, Kim YK. Postoperative complications influence prognosis and recurrence patterns in periampullary cancer. World J Surg 2013;37:2234-2241.

20. Winter JM, Cameron JL, Campbell KA, et al. 1423 Pancreaticoduodenectomies for pancreatic cancer: a single-institution experience. J Gastrointest Surg 2006;10:11991210.

21. Yeo CJ, Sohn TA, Cameron JL, Hruban RH, Lillemoe KD, Pitt HA. Periampullary adenocarcinoma: analysis of 5-year survivors. Ann Surg 1998;227:821-831.

22. Howard TJ, Krug JE, Yu J, et al. A margin-negative Ro resection accomplished with minimal postoperative complications is the surgeon's contribution to longterm survival in pancreatic cancer. J Gastrointest Surg 2006;10:1338-1345.

23. Kang CM, Kim DH, Choi GH, Kim KS, Choi JS, Lee WJ. Detrimental effect of postoperative complications on oncologic efficacy of Ro pancreatectomy in ductal adenocarcinoma of the pancreas. J Gastrointest Surg 2009;13:907914.

24. Ferrone CR, Brennan MF, Gonen M, et al. Pancreatic adenocarcinoma: the actual 5-year survivors. J Gastrointest Surg 2008;12:701-706.

25. Han SS, Jang JY, Kim SW, Kim WH, Lee KU, Park YH. Analysis of long-term survivors after surgical resection for pancreatic cancer. Pancreas 2006;32:271-275. 